\title{
Mapping and review of audiovisual editors and streaming platforms based on collaborative technologies in real time
}

\author{
Brett Stalbaum \\ Speculative Design \\ University of California, San \\ Diego (UCSD) \\ 9500 Gilman Drive, \\ La Jolla, CA \\ stalbaum@ucsd.edu
}

\author{
Cicero I. da Silva \\ Brazil Telehealth Networks \\ Federal University of São Paulo \\ (Unifesp) \\ Rua Sena Madureira, 1500 \\ 4o andar, São Paulo, SP \\ cicero.silva@unifesp.br
}

\author{
Jane de Almeida \\ PPG Education, Art and \\ History of Culture \\ Mackenzie University \\ Rua da Consolação, 930, \\ São Paulo, SP \\ janedealmeida@post.harvard.edu
}

\author{
Maria Amelia Eliseo \\ Department of Computing and Informatics \\ Mackenzie University \\ Rua da Consolação, 930, \\ São Paulo, SP \\ mamelia@gmail.com
}

\author{
Vic Von Poser \\ Mf Art Digital \\ University of the Arts (UAL) \\ 45-65 Peckham Road, London, \\ SE5 8UF \\ totalvic@gmail.com
}

\begin{abstract}
The main goal of this article is to map and review real time video streaming collaborative softwares, in order to analyze platforms that are also able to edit films. Real time high resolution film transmission ( $4 \mathrm{k}, 8 \mathrm{k}$ or above) results in the excess of data generated and, consequently, in a high value for investment in editing, storage and content distribution features. The retrieval and storage of the data (content) becomes complex and expensive. This article aims to design a software review for the use of systems that are applicable to the needs directly related to the digital education field, considering that nowadays the video is an important tool to support teaching and learning processes and needs a better structuring from the educational managers of communication and information technologies.
\end{abstract}

\section{KEYWORDS}

Video processing, video editors, streaming

\section{INTRODUCTION}

This section shows the current scenario regarding editor tools, highlighting their properties related to the ideal features for an online video editor with streaming. Among the existing tools with possibilities of streaming on the web, the best known and most

In: V Workshop "O Futuro da Videocolaboração" (WCT-Video 2018), Salvador, Brasil. Anais do XXIV Simpósio Brasileiro de Sistemas Multimídia e Web: Workshops e Pôsteres. Porto Alegre: Sociedade Brasileira de Computação, 2018. (C)2018 SBC - Sociedade Brasileira de Computação. ISBN: 978-85-7669-435-9. popular are:

a) YouTube; b) Livestream; c) Justin TV; d) XSplit Broadcaster; e) Kaltura; f) Clipbudget; g) Bambuser; h) Wirecast; i) Open Cast

Some stream tools are only dedicated to video features, others stream and store the content for later viewing and editing, if the user wants to edit the material in post-production. Softwares who have video editors allow users to insert clips, mark the beginning and end of a video, and crop unwanted materials. Users can even add a soundtrack.

Below is a brief description of the most popular streaming tools, some of them with editing properties:

a) YouTube is the most widely used streaming model today. The platform allows the editing of the material transmitted after the streaming is finished. However, it does not allow consecutive editing of the transmitted content, i.e. it is not possible to insert audio, cut or even change any content consecutively to the streaming. To carry out a streaming on YouTube you need specific tools that make the connection between the camera, the microphone and the compression system that Youtube will use. For the sake of simplicity, the XSplit Broadcaster tool stands out for its screen capture, streaming and addition of plugins that help in postproduction, as well as being more intuitive and already adapted for Youtube use. Another option is to use Quik ( $\mathrm{G}+$ Hangout on Air), which performs content streaming through the use of the Hangout tool. When the Hangout transmission is finalized, the content can be made available on Youtube.

b) In addition to this tool, there is the Kaltura software, which has both its commercial and its open source 
version with several plug-in options for post-production and web application modules, such as integration with the CMS (Custom Management System) WordPress (VideoPress), with the Joomla CMS, among others.

c) Wirecast Go. The tool enables real-time transmission through mobile devices such as tablets and cell phones. The characteristics of Wirecast are:

c.1) a single camera (front or rear of mobile devices) live;

c.2) is capable of streaming directly to YouTube with just one click;

c.3) add images, logos and graphics to your photo library stream;

c.4) basic editing (resizing and moving graphics);

c.5) unlimited streaming;

d) Skype Qik: video sharing application that allows you to send audio content to your closest friends on your Windows Phone, iPhone or Android;

e) Bambuser: this platform is a good option to use with mobile technologies (cell phones and tablets).

Among the video capture software, a fair option is the Open Broadcaster Software, which is free and interoperable with other systems, such as Kaltura, Adobe Connect and Red5. It is one of the most used systems by gamers to produce their live game streaming via Youtube and Vimeo, among others.

The streaming software system is important because it allows the captured content to be sent through other platforms. In addition, these softwares perform the encoding of the video and audio signal, creating a specific video file that can be transmited through these platforms, such as Youtube, Vimeo, Wevideo, among others. In systems that generate video display protocols, each tool usually generates its own viewer, which makes it easy to display the video stream both on Internet browsers and on the screens of mobile applications. This view is common in applications such as Bambuser, Qik etc. where you can view real-time streaming of content streamed through your mobile (mobile or tablet) applications. In other video systems, such as Youtube, it is possible to send different video streams from the most varied sources, since the compaction and reading systems of these viewers do not contemplate the reading of many formats and, in this sense, it is necessary a software that "reads" the content and presents it. However, these softwares do not allow users to view in real time a timeline with frames being accumulated one after another. The streaming tools usually do not generate separate audio and video files, only allowing post editing of the content. Another lack of this kind of software is that there is no buffering, which would allow the user to decide what they would like to save and to "publish" in real time from the streaming.

\section{SOFTWARES FOR STREAMING}

\subsection{Real time systems}

The table below shows a comparative table between two online video platforms and the respective feeds of video streams supported by these platforms, which means that it is not possible to separate a video stream feed from the Bambuser application, for example, and broadcast it in real time at the same time on Youtube, i.e, Youtube, in this case, accepts a variety of video feeds, not getting dependent only on their own structures of transmission of video feeds

\begin{tabular}{|l|l|l|}
\hline \multicolumn{1}{|c|}{ Software } & \multicolumn{1}{|c|}{ Formats } & \multicolumn{1}{c|}{ Players } \\
\hline Bambuser & MP4 & Bambuser \\
\hline Youtube & $\begin{array}{l}\text { MP4, FLV, } \\
\text { MPEG, MOV, } \\
\text { entre outros }\end{array}$ & $\begin{array}{l}\text { Youtube, XSplit } \\
\text { Broadcaster, Quick } \\
\text { (G+ Hangout) }\end{array}$ \\
\hline
\end{tabular}

Below is a brief list of the most popular streaming tools, some of them with editing properties:
a. Wowza ${ }^{1}$
b. Wirecast ${ }^{2}$
c. Vjdirector $^{3}$
d. WSE Manager ${ }^{4}$
e. Open Cast $^{5}$
f. $23^{6}$

\section{VIDEO EDITORS: STATE OF THE ART}

As soon as there is no real time editing software platform, currently the process of assembling or editing an audiovisual product consists of selecting, ordering, and adjusting the plans or moments in order to achieve the expected result. In this sense, this section reviews the top three most used, by it's market share, video editor softwares and points out the lack of a feature that can be, in the near future, crucial for professionals that work based on live streaming of contents. Before the digital age, one can compare the editing process with writing a text on a typewriter. Before typing, the text should be well outlined, drafted and revised; any subsequent changes to the text on paper were complicated, time-consuming and costly. So were editions of audiovisual products, in which the final product as a whole was thought before going into the post-production process. At the beginning of the cinema, these were physical processes like cutting and gluing of films. With the evolution of technology, recordings began to use magnetic tapes (BETAMAX, VHS), to select and re-record, which resulted in loss of material quality. With these processes there was no opening for error or chance, everything had to be thought out and planned in advance. To this

\footnotetext{
${ }^{1}$ See https://www.wowza.com/

${ }^{2}$ See http://www.telestream.net/wirecast/overview.htm

${ }^{3}$ See http://nagashare.com/vjdirector/

${ }^{4}$ See http://wsemanager.com/

${ }^{5}$ See http://www.opencast.org/

${ }^{6}$ See http://www.23video.com/api/
} 
Mapping and review of audiovisual editors and streaming platforms based on collaborative technologies in real time

type of edition we call "linear editing". With the advent of the computer, "editing software" works with hardwares (Video Cards, HDS), including versions for personal computers. The first system adopted by the market was the hardware and software mix created by NewTek Video Toaster for the Amiga 2000 computer, as shown in Figure 1. The mechanism presented an interface inspired by analog processes, such as the scissor icon representing the cutting tool of the old movie theater moviolas. At the time, this represented a breakthrough in the mobility of images within the video, such as easier erasing or replacing images. It also consisted of a series of effects and tools, transitions and enhancement of low cost images that were once complicated to accomplish. Large concepts presented by this mechanism still resists in modern editing software, such as digital cutting tools and the Timeline concept, as seen in the interface shown in Figure 2. However, it was no longer necessary to think of an integral final product and many experiments can be performed by inserting and removing images within the editing line. This would be the beginning of the concept of "non-linear editing".

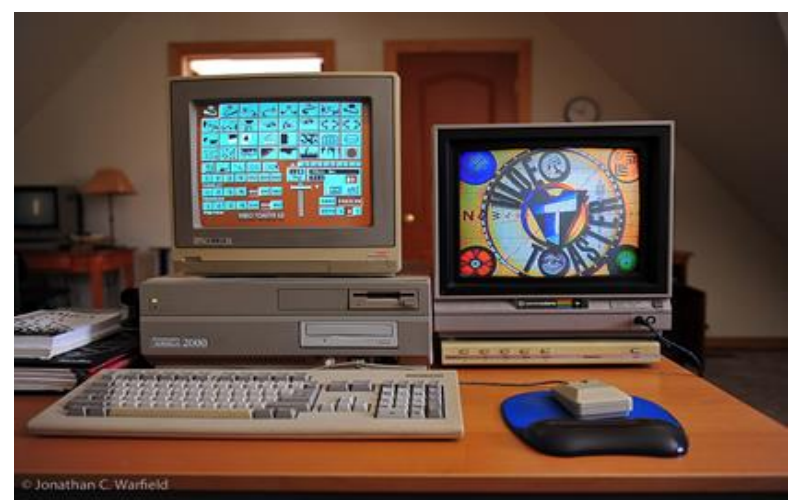

Figure 1: Software and hardware system for video editing in the 1990s, proposed by NewTek Video Toaster.

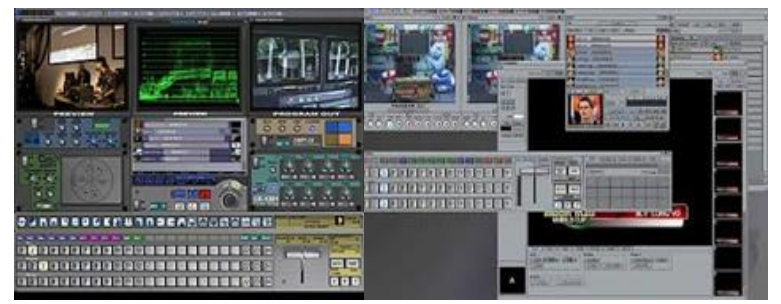

Figure 2: System interface proposed by NewTek Video Toaster 2 .

\subsection{Apple Final Cut}

In the professional universe it is considered the software that has the more sophisticated features and requires more time and practice to be manipulated. At the beginning of the digital age, one of the software preferred by independent audio-visual professionals was Apple Final Cut 7 (Mac OS X only, released in
WebMedia 2018: Workshops e Pôsteres, Salvador, Brasil

2009). It presented better features and a user-friendly interface, with the possibility of greater interaction and manipulation of the material. In 2011, Apple released an update of this software called Final Cut X. In this new version, the software, which was the most used by professionals in the field, has an interface and a range of effects more similar to amateur software mentioned above. Figure 3 shows the interfaces of Final Cut Pro and Final Cut Pro X.
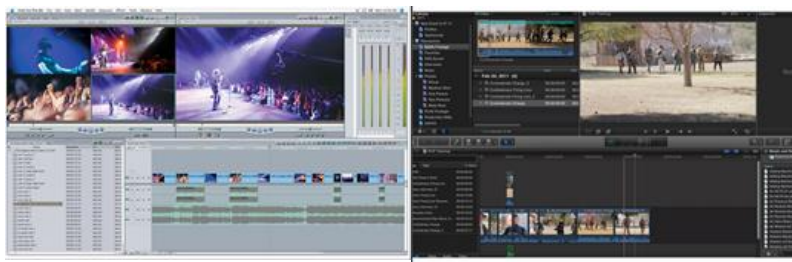

Figure 3: On the left, the interface of Final Cut Pro; on the right, the interface of Final Cut Pro X.

\subsection{Adobe Premiere Pro}

Many users, however, did not adapt to the new presentation of the Final Cut software, because in an attempt to make the software friendly and easy, Apple ended up launching a very similar program with the editing software dedicated to beginners, and in that sense, the professionals finished considering that this version had many limitations. Users were gradually migrating and looking for other software that suited their needs and had a more familiar interface than they were accustomed to. The most sought after was Adobe Premiere Pro (Figure 4), which, with its 2014 update, started to present better and more user-friendly features for professional users, and even improved communication with other Adobe software such as Adobe After Effects, Photoshop and Illustrator. It was also a great advance in the dynamicity of the possibility of importing diverse materials, of various formats and codecs, that operated together in a same timeline, making better use of the time for the publisher, who no longer had to import the videos into the timeline every time they wanted to edit them, or even make a selection of a particular scene.

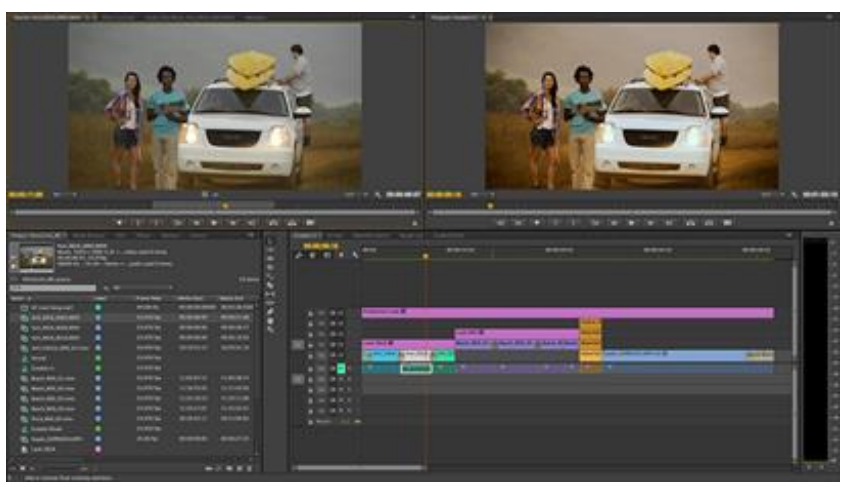


Figure 4: Adobe Premiere Pro interface.

\subsection{Avid}

In the medium and large video production companies, we can highlight the use of the Avid Media Composer tool (Figure 5). Avid is a digital software that still works with the concept of a linear edition. It was released in 1991 as a set of hardware and software, and evolved into a widely used and respected software, especially in large works and by editors/directors accustomed to the beginning of video editing. One advantage of this software is that it generates its own media, so it does not work with the original raw material, which can be copied and saved for other purposes. But, this software is still complicated in terms of a nonlinear video editing, presenting problems with mobility and takes changes. To work with this software, you need to have the thought of the movie as a whole in advance again, as in the analogic film editing process.

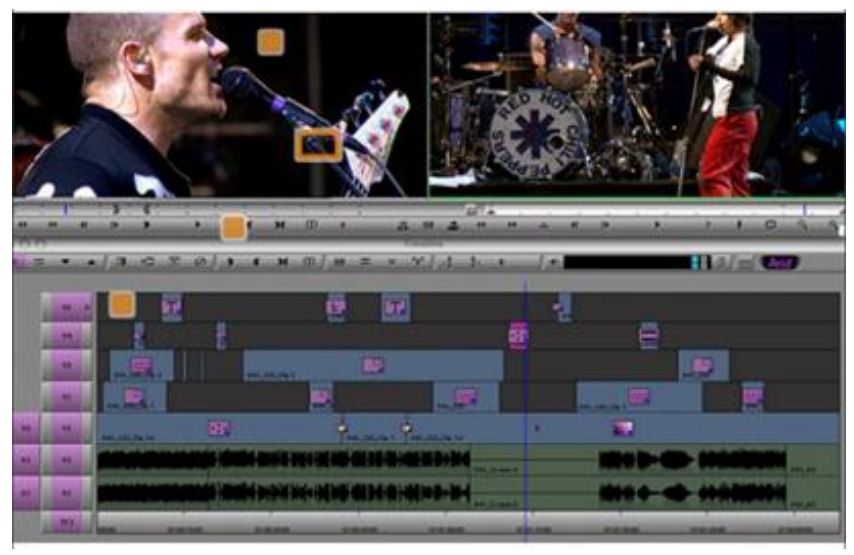

Figure 5: Avid Media Composer interface.

\section{RESULTS AND DISCUSSION}

If the user is a professional or amateur, linear or non-linear, precreated or editable timelines and digital $\mathrm{x}$ analogic editing issues matters more than we could expect. By choosing editing softwares, among the many available, users will always depend on the goals, interests and intents to cut, paste, and manipulate information to build a new meaning for the recorded and scattered images in the digital world. It is important to stress that right now no edition platform can edit online videos in real time, creating a shortage of options for streaming video editors that work in the education and in entertainment fields. On August $30^{\text {th }}, 2018$ PC Mag, one of the most respected magazines in the technology field, ran an article describing the most important video editors based in their market share.
Below is a comparative table of video editors and its features published by PC Mag that corroborates our article review:

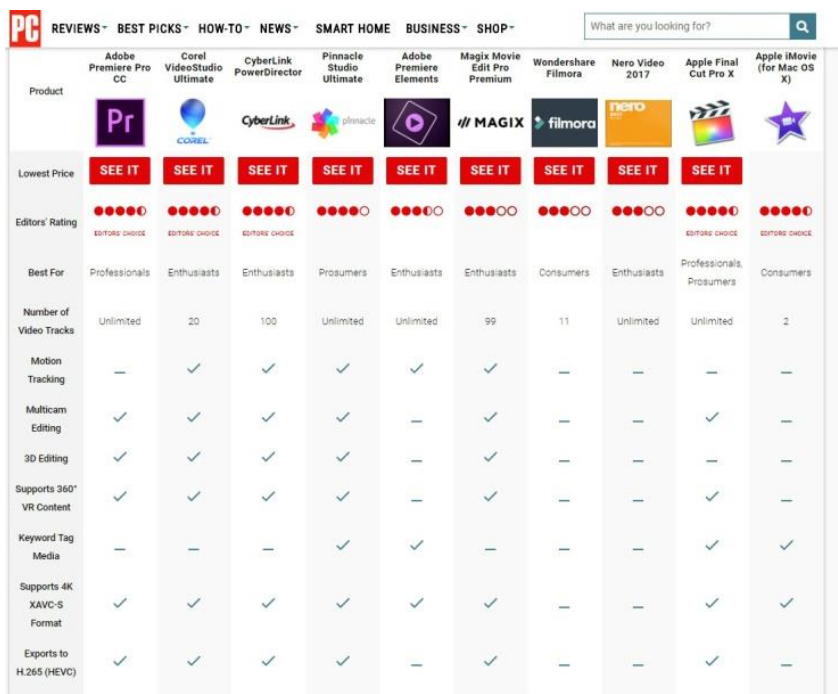

Figure 6: PC Mag table with video editors softwares. [7]

\section{ACKNOWLEDGMENTS}

This work was partially supported by The São Paulo Research Foundation (FAPESP) under its BPE Program (FAPESP grant 17/20434-6).

\section{REFERENCES}

[1] Heide Alvarez. AmLight Consortium Research \& Education Network Helps Transmit FIFA World Cup in $8 \mathrm{~K}$ from Brazil to Japan. Center for Internet Augmented Research and Assessment (CIARA) - Florida International University. Miami-USA. 22 jul 2014. Available online: 〈http://ciara.fiu.edu/images/release_8K-WorkdCup_network.pdf〉. Access: Feb 06, 2017.

[2] Jane de Almeida; Maria Amelia Eliseo; Cicero I. da Silva; Helena Prates; Vic Von Poser; Brett Stalbaum and Nilton Furtado. Sensemaking: A Proposal for a Real-Time on the Fly Video Streaming Platform. Creative Education, v. 07, 2016,p.2515-2523.

[3] Jane de Almeida; Brett Stalbaum; Alfredo Suppia and Cicero I. da Silva Passages on Brazilian scientific cinema. Public Understanding of Science, 2017, Vol. 26(5) 579-595. DOI: https://doi.org/10.1177/0963662516683638 .

[4] Jane de Almeida; Guido Lemos Souza Filho; Carlos Batista; Lucenildo Aquino Junior; Jose Ivan Vilarouca Filho; Manoel Neto; Cicero I. da Silva; Leandro Ciuffo; Iara Machado and Clayton Silva. Integration of Fogo Player and SAGE (Scalable Adaptive Graphics Environment) for 8K UHD Video Exhibition. Journal of Computer and Communications, v. 02, 2014, p. 50-55.7.

[5] Jane de Almeida; Cicero I. da Silva and Thiago Andre (Orgs.). CineGrid: futuros cinemáticos. 1. Ed. São Paulo: CINUSP, 2017.

[6] Gilberto Sobrinho. Cinemas em redes. Tecnologia, estética e política na era digital. Campinas, Papirus, v.1, 2016.

[7] Michael Muchmore. The best video editing software of 2018. PC Mag. Accessed September $1^{\text {st }}$ 2018. Available online: https://www.pcmag.com/article2/0,2817,2397215,00.asp 